\title{
Exchange Coupling of Spin-Crossover Molecules to Ferromagnetic Co Islands
}

Saber Gueddida, ${ }^{\dagger}$ Manuel Gruber, ${ }^{*, \dagger, \$ \S}$ Toshio Miyamachi, ${ }^{\ddagger} / /$ Eric Beaurepaire, ${ }^{\dagger}$ Wulf Wulfhekel, ${ }^{\ddagger I}$ and Mebarek Alouani ${ }^{\dagger}$

†Institut de Physique et de Chimie des Matériaux de Strasbourg (IPCMS), Université de Strasbourg, CNRS UMR 7504, 23 rue du Loess, BP 43, F-67034 Strasbourg Cedex 2, France

Physikalisches Institut, Karlsruhe Institute of Technology, Wolfgang-Gaede-Strasse 1, 76131 Karlsruhe, Germany

${ }^{9}$ Institute of Nanotechnology, Karlsruhe Institute of Technology, 76021 Karlsruhe, Germany

${ }^{\S}$ Present address: IEAP, Christian-Albrechts-Universität zu Kiel, 24098 Kiel, Germany

"Present address: The Institute for Solid State Physics (ISSP), The University of Tokyo, Kashiwa 277-8581, Japan 


\section{Calculated supercell}

The experimental substrate, i.e. Co bilayer islands on $\mathrm{Cu}(111)$, is too large to be correctly calculated. Moreover, the experimental size and shape of the Co islands are not constant. We therefore approximated the experimental system to the following supercell:

- $\quad 3$ atomic layers of $f c c \mathrm{Cu}$ with a lattice parameter of $3.6 \AA$. Each atomic layer is composed of 48 atoms.

- $\quad 2$ atomic layers of $f c c$ Co using the same lattice parameter than for $\mathrm{Cu}$.

- One Fe-phen molecule composed of 51 atoms ${ }^{1}$.

The supercell is therefore composed of a total of 291 atoms (see Fig. S1). We used $3 \mathrm{~nm}$ vacuum to separate the periodic supercell. All atoms of the molecule and that of the first Co atomic layer are relaxed, but the four lower atomic layers are kept fixed. The convergence criterion for the relaxation was set to $10^{-5} \mathrm{Ry} / \AA$.

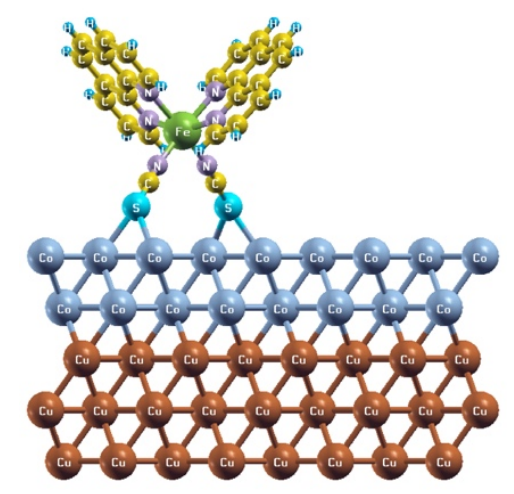

Fig. S1. Calculated (relaxed) supercell of HS Fe-phen on $\mathrm{Co} / \mathrm{Cu}(111)$.

\section{Details about molecular adsorption}

We show in Fig. S1 the result of the relaxed structure of the molecule on the $\mathrm{Co} / \mathrm{Cu}(111)$ substrate. The two sulfur atoms of HS and LS Fe-phen are in center positions. The equilibrium distance between the Fe-phen molecule and the surface calculated using GGA+U+vdW is 1.84 $\AA$ in HS and $2.02 \AA$ in LS state. The corresponding calculated adsorption energies of both HS and LS configurations are respectively $-3.04 \mathrm{eV}$ and $-3.18 \mathrm{eV}$.

\section{Spin-polarized conductance deduced from a Jullière-based model}

We estimated a spin-dependent conductance from Jullière's model using the calculated DOS of adsorbed HS Fe-phen and the calculated DOS of an ideal magnetic STM tip.

The idealized STM tip is composed of three atomic layers of $f c c \mathrm{Co}(111)$ and 20 additional Co atoms that form the tip apex (see Fig. S2). The Co atoms of the tip apex are relaxed. In Fig. S2, 
we additionally show the calculated spin-dependent DOS of the model tip. As expected for a ferromagnetic material, the DOS is quite different for spin up and spin down electrons.
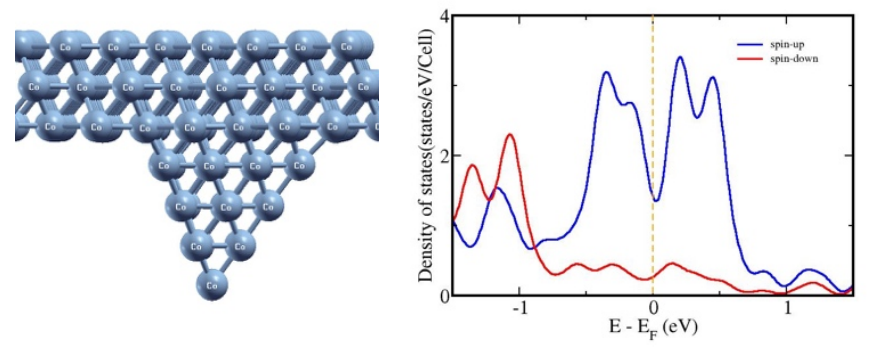

Fig. S2. (left) Schematic of the STM tip used for the calculations. (right) DOS of the model STM tip calculated using GGA+U+vdW.

In order to evaluate the spin-dependent conductance of the system composed of the tip and the adsorbed molecule, we extended Jullière's model ${ }^{2}$ initially defined the Fermi level to the entire energy range. The conductance of the system for a parallel magnetic configuration is then given by:

$$
G_{P}\left(E-E_{F}\right)=\rho_{\text {mol }}^{\uparrow}\left(E-E_{F}\right) * \rho_{\text {tip }}^{\uparrow}\left(E-E_{F}\right)+\rho_{\text {mol }}^{\downarrow}\left(E-E_{F}\right) * \rho_{\text {tip }}^{\downarrow}\left(E-E_{F}\right)
$$

where $\rho_{\text {mol } / \text { tip }}^{\text {spin }}\left(E-E_{F}\right)$ refers to the calculated DOS of the adsorbed Fe-phen molecule or of the Co tip with spin up or down at the energy $E-E_{F}$. Similarly, the conductance for an antiparallel magnetic configuration is:

$$
G_{A P}\left(E-E_{F}\right)=\rho_{m o l}^{\uparrow}\left(E-E_{F}\right) * \rho_{\text {tip }}^{\downarrow}\left(E-E_{F}\right)+\rho_{m o l}^{\downarrow}\left(E-E_{F}\right) * \rho_{t i p}^{\uparrow}\left(E-E_{F}\right)
$$

\section{Magnetization density of the LS state}

In the main manuscript, we show the calculated magnetization density of HS Fe-phen on $\mathrm{Co} / \mathrm{Cu}(111)$. We performed the same calculations on LS Fe-phen and we show the result in Fig. S3. Upon adsorption on $\mathrm{Co} / \mathrm{Cu}(111)$, the Fe center of LS Fe-phen acquires a net magnetic moment of $0.009 \mu \mathrm{B}$ that is ferromagnetically coupled to the $\mathrm{Co} / \mathrm{Cu}(111)$ substrate. 


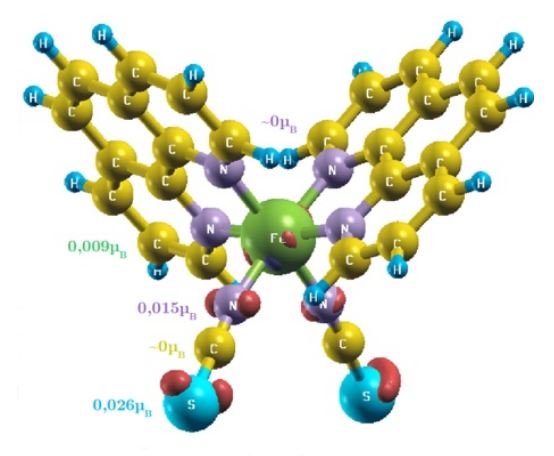

Fig. S3. Calculated magnetization density of LS Fe-phen using the GGA+U+vdW method. The positive magnetization density is red, and the negative is blue. The values of the magnetic moments of iron, nitrogen, carbon and sulfur are also indicated.

\section{Spin-polarized scanning tunneling spectra of the Co islands}

In Fig. 3 of the manuscript, we show STS spectra acquired over two HS Fe-phen molecules, which were hosted on two Co islands with opposite magnetization. For completeness, we show the STS spectra acquired over these two Co islands in Fig. S4.

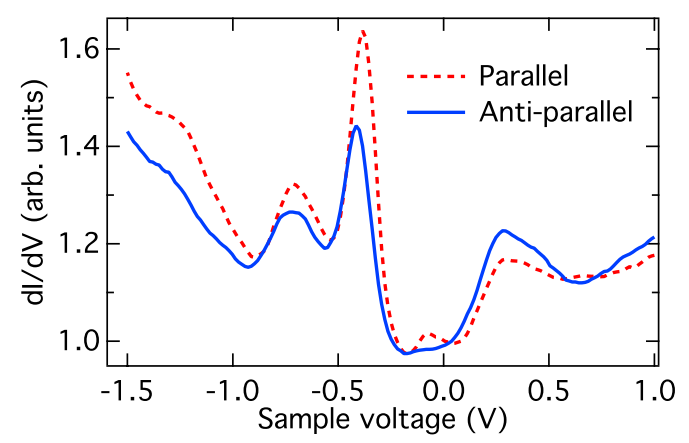

Fig. S4. Experimental dl/dV spectra acquired over two Co islands with opposite magnetization orientation. The definition of parallel and anti-parallel alignment of the tip and Co-island magnetizations is defined in the manuscript.

\section{Fe-phen on $\mathrm{Cu}(111)$}

In Fig. S5, we show topographies of Fe-phen deposited on bare $\mathrm{Cu}(111)$. The two-lobe shape of Fe-phen on $\mathrm{Cu}(111)$ (see Fig. S5(a)) is essentially similar to that of Fe-phen on $\mathrm{Cu}(100)^{3}$. Furthermore, the molecules stay isolated and the distribution of the molecules over the surface is rather homogeneous (see the large-scale topography in Fig. S5(b)). Yet, when Fephen is deposited on $\mathrm{Co} / \mathrm{Cu}(111)$, we do not observe Fe-phen molecules over the $\mathrm{Cu}(111)$ area (see Fig. 1(b)). Instead, the Fe-phen molecules are only on the Co islands and presumably around the Co islands. We believe that this is due to the higher reactivity of the molecules with Co. In turn, the higher reactivity of the molecules with Co would explain the differences in the topographies of Fe-phen on $\mathrm{Cu}(111)$ and on $\mathrm{Co}$. 


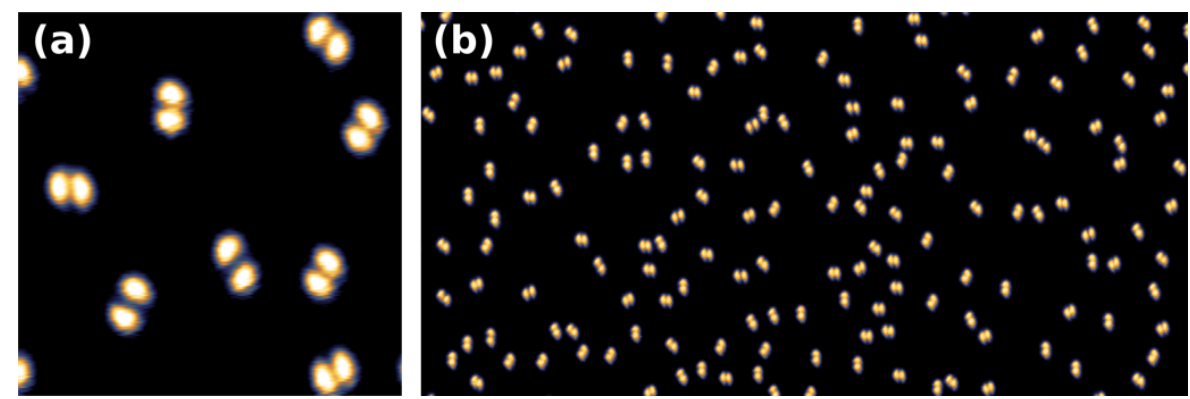

Figure S5. Topographies of Fe-phen on bare $\mathrm{Cu}(111)$. Image sizes are (a) $14.1 \mathrm{~nm} \times 14.1 \mathrm{~nm}$ and (b) $110 \mathrm{~nm} \times 55 \mathrm{~nm}$.

\section{References}

(1) Gueddida, S.; Alouani, M. Spin Crossover in a Single Fe(phen)_\{2\}(NCS)_\{2\} Molecule Adsorbed onto Metallic Substrates: An Ab Initio Calculation. Phys. Rev. B 2013, 87 (14), 144413.

(2) Julliere, M. Tunneling between Ferromagnetic Films. Phys. Lett. A 1975, 54 (3), 225-226.

(3) Miyamachi, T.; Gruber, M.; Davesne, V.; Bowen, M.; Boukari, S.; Joly, L.; Scheurer, F.; Rogez, G.; Yamada, T. K.; Ohresser, P.; et al. Robust Spin Crossover and Memristance across a Single Molecule. Nat. Commun. 2012, 3, 938. 